\title{
The Gauss-Landau-Hall problem on Riemannian surfaces
}

\author{
Manuel Barros ${ }^{1}$, José L. Cabrerizo ${ }^{2}$, Manuel Fernández ${ }^{2}$ \\ and Alfonso Romero ${ }^{1}$ \\ ${ }^{1}$ Departamento de Geometría y Topología, Facultad de Ciencias \\ Universidad de Granada, 18071-Granada, Spain. \\ E-mail addresses: mbarros@ugr.es, aromero@ugr.es \\ ${ }^{2}$ Departamento de Geometría y Topología, Facultad de Matematicas \\ Universidad de Sevilla, 41012-Sevilla, Spain. \\ E-mail addresses: jaraiz@us.es, mafernan@us.es
}

\begin{abstract}
We introduce the notion of Gauss-Landau-Hall magnetic field on a Riemannian surface. The corresponding Landau-Hall problem is shown to be equivalent to the dynamics of a massive boson. This allows one to view that problem as a globally stated, variational one. In this framework, flowlines appear as critical points of an action with density depending on the proper acceleration. Moreover, we can study global stability of flowlines. In this equivalence, the massless particle model correspond with a limit case obtained when the force of the Gauss-Landau-Hall increases arbitrarily. We also obtain new properties related with the completeness of flowlines for a general magnetic fields. The paper also contains new results relative to the Landau-Hall problem associated with a uniform magnetic field. For example, we characterize those revolution surfaces whose parallels are all normal flowlines of a uniform magnetic field.
\end{abstract}

\section{From a classical picture to a general setting}

Classically, the Landau-Hall problem consists of the motion study of a charged particle in the presence of a static magnetic field, $H$. In this setting, free of any electric field, a particle, of charge $e$ and mass $m$, evolves with velocity $v$ satisfying the Lorentz force law, [1],

$$
\frac{d P}{d t}=\frac{e}{c} v \times H
$$

where $c$ denotes the light speed, $P=\left(\epsilon / c^{2}\right) v$ stands for the momentum of the particle, and $\epsilon=m c^{2}\left[1-\left(\|v\|^{2} / c^{2}\right)\right]^{-1 / 2}$ is its energy. Since $d P / d t$ is orthogonal 
to $P$, then $(d / d t)\left(\|P\|^{2}\right)=0$. This implies the constancy of both $\|v\|$ and $\epsilon$. Assume $H$ is stationary, i.e., $H$ is a time-independent vector of the Euclidean space $\mathbb{R}^{3}$. With the choice of a suitable orthonormal reference system, we may assume that $H=h(0,0,1)$, for some $h \in \mathbb{R}$. In this framework, we have

$$
\frac{d}{d t} v_{1}(t)=\omega v_{2}(t), \quad \frac{d}{d t} v_{2}(t)=-\omega v_{1}(t), \quad \frac{d}{d t} v_{3}(t)=0
$$

where $\omega=(e h c) / \epsilon$ is constant. Then

$$
x_{1}(t)=x_{1}^{0}+r \sin (\omega t+\alpha), \quad x_{2}(t)=x_{2}^{0}+r \cos (\omega t+\alpha), \quad x_{3}(t)=x_{3}^{0}+v_{3}^{0} t,
$$

where $r=\|v\| / \omega$. In particular, if $v_{3}^{0}=0$, then the particle describes a circle in the plane $x_{3}=x_{3}^{0}$, with center $\left(x_{1}^{0}, x_{2}^{0}, x_{3}^{0}\right)$ and radius $r$. Now, in this plane we consider the 2-form $F$ defined by $F(X, Y)=\varepsilon<X \times Y, H>$, where $\varepsilon= \pm 1$ is the sign of $h / \omega$. It is clear that $F$ is covariantly constant, and therefore it is a constant multiple of the area element, indeed $F=\varepsilon h d x_{1} \wedge d x_{2}$. Now, consider the metric $g$ on the plane defined by $g:=\varepsilon(h / \omega) g^{0}$, where $g^{0}=<,>$ denotes the Riemannian metric on the plane induced by the usual one of $\mathbb{R}^{3}$. Define the operator $\Phi, g$-equivalent to $F$, by $g(\Phi(X), Y)=F(X, Y)$. Then, the Lorentz force law can be expressed in terms of this form by

$$
\frac{d}{d t} v(t)=\Phi(v(t))
$$

This approach to the classical picture can be obviously extended to a more general setting. In fact, it seems natural to define a magnetic field on a $n(\geq 2)$ dimensional Riemannian manifold $(M, g)$, as a closed 2-form $F$ on $M$. The Lorentz force of a magnetic background $(M, g, F)$ is defined to be the skew-symmetric operator, $\Phi$, given by

$$
g(\Phi(X), Y)=F(X, Y)
$$

for any couple of vector fields $X, Y$ on $M$. Let us remark that $\Phi$ is metrically equivalent to $F$, so no information is lost when $\Phi$ is considered instead $F$. In classical terminology, it is said that $\Phi$ is obtained from $F$ by raising its second index, and $\Phi$ and $F$ are then said to be physically equivalent. On the other hand, there exists another operator $\Phi^{\prime}$ defined from $F$ via $g$ in a similar way, namely $g\left(X, \Phi^{\prime}(Y)\right)=F(X, Y)$, but it is easily seen that $\Phi^{\prime}=-\Phi$. So, the choice from among $\Phi$ or $\Phi^{\prime}$ to represent $F$, using $g$, is not relevant. Along this paper, we will use $\Phi$ to denote the Lorentz force induced from $(M, g, F)$.

A (smooth) curve $\gamma$ in $(M, g)$ is called a flowline of the dynamical system associated with the magnetic field $F$ (or simply a flowline of $F$, or a magnetic curve of $(M, g, F)$ ), if its velocity vector field, $\gamma^{\prime}$, satisfies the following (Landau-Hall) differential equation,

$$
\nabla_{\gamma^{\prime}} \gamma^{\prime}=\Phi\left(\gamma^{\prime}\right)
$$


where $\nabla$ is the Levi-Civita connection of $g$ [compare with Eq. (11)].

For the trivial magnetic field, $F=0$, the case without the force of a magnetic field, magnetic curves correspond with the geodesics of $(M, g)$. As it is well known, they are nicely characterized as critical points of an energy action and so they represent the trajectories for free fall particles (moving under the influence of only gravity). In the general case, however, magnetic flows are important examples of dynamical systems on Riemannian manifolds whose flowlines, being the trajectories of charged particles in (non trivial) magnetic fields, are not geodesics (Proposition 2.1) but, as we will see later, they are closely related with the Riemannian structure.

Nevertheless, the magnetic curves of $(M, g, F)$ can be also viewed, at least locally, as the solutions of a variational principle. In fact, let $U$ be an open subset of $M$ where $F=d \omega$ for some potential 1-form $\omega$ (this open subset could be the whole $M$ when $\left.H^{2}(M)=0\right)$. For any two fixed points $p, q \in U$, we consider the space $\Gamma_{p q}$ of smooth curves in $U$ that connect these two points. Now, we choose the action $\mathcal{L H}: \Gamma_{p q} \rightarrow \mathbb{R}$ defined by

$$
\mathcal{L} \mathcal{H}(\gamma)=\frac{1}{2} \int_{\gamma} g\left(\gamma^{\prime}, \gamma^{\prime}\right) d t-\int_{\gamma} \omega\left(\gamma^{\prime}\right) d t
$$

The tangent space of $\Gamma_{p q}$ in $\gamma$ is made up of the smooth vector fields, $V$, along $\gamma$ that vanish at the end points $p, q \in U$. An standard computation involving integration by parts allows one to compute the first variation of this action to be

$$
\delta(\mathcal{L H})(\gamma)[V]=-\int_{\gamma} g\left(\nabla_{\gamma^{\prime}} \gamma^{\prime}-\Phi\left(\gamma^{\prime}\right), V\right) d t
$$

As a consequence, we get

$$
\delta(\mathcal{L H})(\gamma)[V]=0 \text {, for any } V \in T_{\gamma} \Gamma_{p q} \text { if and only if } \gamma \text { is a solution of (LH). }
$$

This argument shows that the differential equation (LH) is indeed the Euler-Lagrange equation associated with the functional $\mathcal{L} \mathcal{H}$.

However, it seems natural to realize the old idea of characterizing magnetic curves from a global variational principle. In other words, to obtain the magnetic trajectories of $(M, g, F)$ as solutions of a variational problem that neither it does not involves any local potential nor it does not constraint the topology of $M$. This is, in general, an interesting open problem. One of the main aim of this paper is just to solve it for certain magnetic fields on surfaces.

To be precise, we introduce the notion of a Gauss-Landau-Hall magnetic field (in brief, GMF) on an oriented Riemannian surface $(M, g)$. First, we do it in the natural context that surfaces are immersed in Euclidean space $\mathbb{R}^{3}$ using the Gauss map. However, we notice that the notion of GMF is absolutely intrinsic so it can be considered on surfaces even if they are not regarded in $\mathbb{R}^{3}$. Then, we are able to obtain an amazing result which characterizes the normal flowlines of a GMF as the solutions of a variational principle globally stated. Therefore, those flowlines appear as critical points of an action whose Lagrangian density involves the proper acceleration of particles (relativistic particles with rigidity of order one, in the sense 
of Plyushchay, [2, 3]). A priori, these actions describe a massive relativistic boson. However, massless particles with arbitrary helicity are obtained as a limit case, just when the Lorentz force of the GMG increases arbitrarily.

Other details on the paper are the following. We first provide in Section 2 an analysis of the existence, uniqueness, extendibility and completeness of the magnetic curves associated with a given $(M, g, F)$. Section 3 deals with uniform magnetic fields on Riemannian surfaces, while the particular case when $(M, g)$ is a revolution surface is studied in Section 4. In Section 5, a one-parameter family $\mathcal{F}_{m}$ of functionals is considered on an appropriate space of curves $\Lambda$ in the surface. The Euler-Lagrange equation associated to the variational problem is then obtained. In Section 6 we define a Gauss-Landau-Hall magnetic field on a surface, first in $\mathbb{R}^{3}$, and then in general. In this section, we obtain the main result, Theorem 6.1, which asserts that the normal flowlines of a GMF coincide with the critical points of the appropriate functional $\mathcal{F}_{m}$. Stability of the field equation solutions is also studied. In Section 7 , we show a characterization theorem for those revolution surfaces whose parallels are all normal magnetic curves associated to a GMF. We close the section studying some particular examples.

\section{Completeness of magnetic curves and more}

An early property of the magnetic curves is the following conservation's law. Particles evolve with constant speed, and so constant energy, along the magnetic trajectories

$$
\frac{d}{d t} g\left(\gamma^{\prime}, \gamma^{\prime}\right)=2 g\left(\Phi\left(\gamma^{\prime}\right), \gamma^{\prime}\right)=0
$$

In particular, a magnetic curve $\gamma$ is said to be normal if it has unit energy, i.e., $\left\|\gamma^{\prime}\right\|^{2} \equiv 1$

The existence and uniqueness of geodesics, remains true when one considers magnetic curves. Thus, for each $p \in M$ and $v \in T_{p} M$ there is exactly one inextendible (i.e., maximal) magnetic curve, $\gamma:(-a, a) \longrightarrow M$, of $(M, g, F)$ with $\gamma(0)=p$ and $\gamma^{\prime}(0)=v$, (see for instance [4], p. 91). Since the proof of this result does not make use neither the definiteness of $g$ nor the skew-symmetry of $\Phi$, one has a present determines the future type result for an indefinite metric, Lorentzian in particular, and for any smooth operator. Even more, the result also works for solutions of a differential equation that extends that of Landau-Hall in the following terms [5],

$$
\nabla_{\gamma^{\prime}} \gamma^{\prime}=\Phi\left(\gamma^{\prime}\right)+X \circ \gamma
$$

where $X$ is a vector field on a semi-Riemannian manifold. This setting includes the important case in Mechanics where $X=-\nabla V$, and $V$ standing for smooth function on $M,([6]$, Proposition 3.7.4).

Nevertheless, the well known homogeneity result for geodesics, works quite different in non trivial magnetic fields. Therefore, if $\gamma$ is the inextendible magnetic curve of $(M, g, F)$ determined from the initial data $(p, v)$, the curve $\beta$, defined by $\beta(t)=\gamma(\lambda t), \lambda \in \mathbb{R} \backslash\{0\}$, is a magnetic trajectory of $(M, g, \lambda F)$ and also, when 
$\lambda>0$, of $(M,(1 / \lambda) g, F)$, in both cases determined from initial data $(p, \lambda v)$. Furthermore, the whole families of magnetic curves of $(M, g, F)$ and $(M, \lambda g, \lambda F)$ coincides, for any constant $\lambda>0$. Consequently, we have

Proposition 2.1 Let $F$ be a non trivial magnetic field on a Riemannian manifold, $(M, g)$. Then, there exists no affine connection on $M$ whose geodesics are the magnetic curves of $(M, g, F)$.

A magnetic field $(M, g, F)$ with Lorentz force $\Phi$, provides, in a similar way as in [7, Prop. 3.28, with a unique vector field $Q_{\Phi}$ on the tangent bundle $T M$. This is defined to have integral curves being the lifting to $T M$ of the magnetic curves, that is, $t \mapsto\left(\gamma(t), \gamma^{\prime}(t)\right)$, where $\gamma$ is a magnetic curve of $(M, g, F)$ (compare with [8]). Certainly this vector field is nothing but the geodesic flow when $F=0$. Once more, neither the definiteness of $g$ nor the skew-symmetry of $\Phi$ is needed to define $Q_{\Phi},[5]$. On the other hand, the fact that any integral curve of $Q_{\Phi}$ is the velocity of its projection on $M$, allows us to think of $Q_{\Phi}$ as a nice example of the classically so-called second order differential equation on $M$. Because the comment previous to Proposition 2.1. $Q_{\Phi}$ is not an spray, in general.

A dynamical system with complete trajectories is often thought in Physics to be persisting eternally. But in many circumstances one has to deal with incompleteness. So, because of its importance, we next give criteria to assert when it holds true. An important tool to study the completeness of the inextendible magnetic curves, i.e., under what assumptions all the inextendible magnetic curves are defined on all $\mathbb{R}$, is the vector field $Q_{\Phi}$. By using Lemma 1.56 in [7], it is easily seen the following result.

Proposition 2.2 Let $(M, g)$ be a Riemannian manifold, $F$ a magnetic field on $M$ and $\gamma:[a, b) \longrightarrow M, a<b$, a magnetic curve of $F$. The following are equivalent:

(a) $\gamma$ is extendible to $b$ as a magnetic curve.

(b) There exists a sequence $\left\{t_{n}\right\} \longrightarrow b, t_{n} \in[a, b)$ such that the sequence of velocities $\left\{\gamma^{\prime}\left(t_{n}\right)\right\}$ converges in $T M$.

Accordingly, a magnetic curve $\gamma:(a, b) \longrightarrow M, a, b \in \mathbb{R}, a<b$, of $(M, g, F)$ can be extended to some open interval $I,(a, b) \subset I$, if and only if $\gamma(a, b)$ is contained in a compact subset of $M$. Therefore, we get (compare with Theorem 2.1.18 in [6])

Proposition 2.3 Let $\gamma$ be an inextendible magnetic curve of $(M, g, F)$ such that $\gamma(a, b)$ lies in a compact subset of $M$, for every finite interval $(a, b)$ in its domain. Then, $\gamma$ must be complete.

In particular, if $M$ is assumed to be compact, then we get that any inextendible magnetic curve of $(M, g, F)$ must be complete. This fact can be also obtained as a consequence of Corollary 2.4, and it will be stated in Remark 2.5 (a), from a different approach. 
Now, let $\gamma:[a, b) \longrightarrow M$ be a magnetic curve. Its length $L(\gamma)$ satisfies $L(\gamma) \leq$ $(b-a) \sqrt{e}$, where $e$ is the (constant) energy of $\gamma$. For each $t \in[a, b)$, the distance between $\gamma(a)$ and $\gamma(t)$ satisfies $d(\gamma(a), \gamma(t)) \leq L\left(\left.\gamma\right|_{[a, t]}\right) \leq(b-a) \sqrt{e}$, which shows that $\gamma([a, b))$ is contained in the closed metric ball $B$ centered at $\gamma(a)$ and with radius $(b-a) \sqrt{e}$. Therefore,

$$
\gamma^{\prime}([a, b)) \subset\{(p, v) \in T M: p \in B, g(v, v)=e\} \subset T M .
$$

Then, we have,

Corollary 2.4 Let $F$ be any magnetic field on a geodesically complete Riemannian manifold $(M, g)$. Then, all the inextendible magnetic curves of $(M, g, F)$ are complete.

Proof. If $(M, g)$ is assumed to be geodesically complete, then the Hopf-Rinow theorem implies that $B$ must be compact. Hence $\{(p, v) \in T M: p \in B, g(v, v)=e\}$ is a compact subset of $T M$. Take now a sequence $\left\{t_{n}\right\} \longrightarrow b, t_{n} \in[a, b)$, then $\left\{\gamma^{\prime}\left(t_{n}\right)\right\}$ lies in a compact subset of $T M$. So, by passing to a subsequence of $\left\{t_{n}\right\}$, we are under the assumption (b) of Proposition [2.2, concluding that $\gamma$ is extendible to $b$ as a magnetic curve.

\section{Remark 2.5}

(a): If $M$ is assumed to be compact (therefore $(M, g)$ is geodesically complete for any Riemannian metric $g$ on $M$ ), then we can give an alternative proof of Corollary 2.4. In fact, the previous conservation's law [Eq. (44)] for the length of velocity vectors of magnetic curves, implies that the vector field $Q_{\Phi}$ on $T M$ can be restricted to each spherical tangent bundle $U_{e} M=\{(p, v) \in T M$ : $g(v, v)=e\} \subset T M, e>0$. But $U_{e} M$ is compact whenever $M$ is compact, and hence the restriction of $Q_{\Phi}$ to $U_{e} M$ is a complete vector field. This proves that all the inextendible magnetic curves of $F$ are complete.

(b): Proposition 2.1] has shown a remarkable difference between magnetic curves and geodesics. The following non-connecteness fact complement that result. Let us consider the unit 2-sphere $\mathbb{S}^{2}(1)$ endowed with its standard round metric $g$, and let $F$ be the magnetic field $F=\mu \Omega_{2}$, where $\Omega_{2}$ is the area 2-form and $\mu \in \mathbb{R}, \mu \neq 0$. As we will show later (see the comment after Proposition [3.2), the associated magnetic curves with energy $e$ are circles on $\mathbb{S}^{2}(1)$ with radius $r=\left[1+\left(\mu^{2} / e\right)\right]^{-1 / 2}$. Then, as $r<1$, any two antipodal points can not be connected by a magnetic curve of $\left(\mathbb{S}^{2}, g, F\right)$. Moreover, for any $p \in \mathbb{S}^{2}$, all the inextendible magnetic curves $\gamma$ of $\left(\mathbb{S}^{2} \backslash\{-p\}, g, F\right)$ such that $\gamma(0)=p$ are complete.

(c): Let $(M, g)$ be a Riemannian manifold where $g$ is an incomplete metric. If $F$ is a magnetic field on $(M, g)$, then there exists a pointwise conformal metric $f^{2} g$ such that the inextendible magnetic curves of $\left(M, f^{2} g, F\right)$ are complete. In fact, there exists $f \in C^{\infty}(M), f>0$, such that $f^{2} g$ is geodesically complete, 9]. Therefore, Proposition 2.4 gives that the magnetic curves of $\left(M, f^{2} g, F\right)$ are complete. 
(d): It should be observed that the closedness assumption on the 2-form $F$ in Proposition 2.4 was not used. On the other hand, the skew-symmetry of the tensor field $F$ has played a crucial role [recall the conservation's law (44)]. In fact, consider the tensor field $F=-2 x d x^{2}$ on the Euclidean plane $\left(\mathbb{R}^{2}, g^{0}=d x^{2}+d y^{2}\right)$. If $\Phi$ denotes the operator defined from $F$ using Eq. (2), then $\Phi(\partial / \partial x)=-2 x(\partial / \partial x)$ and $\Phi(\partial / \partial y)=0$. Therefore, $\gamma(t)=(x(t), y(t))$ satisfies Eq. (LH) if and only if $x^{\prime \prime}(t)+2 x(t) x^{\prime}(t)=0$ and $y^{\prime \prime}(t)=0$. So, $\gamma(t)=(1 / t, t)$ is an inextendible incomplete trajectory of $\left(\mathbb{R}^{2}, g^{0}, F\right)$.

(e): Finally, let us point out that Proposition 2.4 cannot be also extended to the indefinite case. In fact, consider $\mathbb{R}^{2}$ endowed with the Lorentzian metric $g_{L}=d x^{2}-d y^{2}$, and define the magnetic field $F=-x d x \wedge d y$. A curve $(x(t), y(t))$ is a magnetic curve of $\left(\mathbb{R}^{2}, g_{L}, F\right)$ if and only if it satisfies $x^{\prime \prime}(t)=x(t) y^{\prime}(t), \quad y^{\prime \prime}(t)=x(t) x^{\prime}(t)$. Then, $\gamma(t)=(2 / t,-2 / t)$ is an inextendible magnetic curve which is defined on $(0, \infty)$.

\section{Uniform magnetic fields}

From now on, $M$ will be an oriented Riemannian surface with standard complex structure $J$, and area element $\Omega_{2}$ so that $\Omega_{2}(X, J X)=1$ for any unit vector field $X$ in $M$.

Given a curve $\gamma$ in $M$ such that $g\left(\gamma^{\prime}, \gamma^{\prime}\right)=e>0$ is constant, its Frenet apparatus is $\left\{T=(1 / \sqrt{e}) \gamma^{\prime}, N=J T\right\}$. If $\kappa$ denotes the curvature function, we have the following well-known Frenet equations

$$
\nabla_{\gamma^{\prime}} T=\kappa \sqrt{e} N, \quad \nabla_{\gamma^{\prime}} N=-\kappa \sqrt{e} T
$$

Obviously, any magnetic field on a surface, $M$, is determined from a smooth function, $f$ (the strength), by $F=f \Omega_{2}$. Therefore, the matrix of $\Phi$ in any orthonormal frame, $\{X, J X\}$ is given by

$$
\left(\begin{array}{rr}
0 & -f \\
f & 0
\end{array}\right)
$$

In particular, along a magnetic curve $\gamma$ of $(M, g, F)$, with energy $e$, and relative to its Frenet frame, the Lorentz force is obtained to be

$$
\left(\begin{array}{rr}
0 & -\kappa \sqrt{e} \\
\kappa \sqrt{e} & 0
\end{array}\right) .
$$

Therefore, we get,

Proposition 3.1 The curvature of the magnetic curves with energy e is given by $\kappa=f / \sqrt{e}$. So, the curvature of the normal magnetics curves completely determines the Lorentz force, i.e., $f=\kappa$ along these flowlines.

A parallel magnetic field $F$, i.e., a magnetic field with constant strength $f=\mu$, is called a uniform magnetic field. This class of magnetic fields has been extensively 
considered in the literature from different points of view $([8,[10]-[16]$, etc.). The geometric partner of the Landau-Hall problem, for uniform magnetic fields, is nothing but the computation of curves with constant curvature. To be precise, we have,

Proposition 3.2 Let $F=\mu \Omega_{2}$ be a uniform magnetic field, with constant strength $\mu$, on a Riemannian surface $(M, g)$. A curve $\gamma$ in $M$, with constant energy $e$, is a magnetic curve of $(M, g, F)$ if and only if it has constant curvature $\kappa=\mu / \sqrt{e}$.

On surfaces of constant Gauss curvature, the feature of the normal flowlines of a non-trivial uniform magnetic field $F=\mu \Omega_{2}$ is well-known for any uniform magnetic field. On the Euclidean plane, $\mathbb{R}^{2}$, they are circles with radius $1 /|\mu|$. On the 2 -sphere of radius $r, \mathbb{S}^{2}(r)$, flowlines with energy $e$ are circles with radius $(r \sqrt{e}) / \sqrt{e+r^{2} \mu^{2}}$ $(<r)$. In these two backgrounds, the flowlines are always closed.

On the other hand, the situation in a hyperbolic plane is quite different. Let $\mathbb{H}^{2}(-G)$ be the upper half-plane (in $\mathbb{R}^{2}$ ) endowed with the Lobatchevski metric of curvature $-G, G>0$, that is, the Poincaré plane. We use Proposition 3.2 joint the basic knowledge of the curves of constant curvature in $\mathbb{H}^{2}(-G)$ (see any basic text of Riemannian geometry) to make trivial the following description of the flowlines which is due to A. Comtet, [11, and has been mentioned along a large list of references. The behaviour of normal magnetic curves changes according to the ratio between the strength, $\mu$, and the curvature of $\mathbb{H}^{2}(-G)$. Namely,

- If $|\mu| / \sqrt{G}>1$, then the trajectories are geodesic circles, and therefore they are closed curves.

- If $|\mu| / \sqrt{G} \leq 1$, then the trajectories are non-closed curves which intersect the boundary line, $\partial \mathbb{H}^{2}(-G)$, of the upper half-plane. In particular, they are tangent to this boundary, and so they are horocycles when $|\mu|=\sqrt{G}$.

\section{Remark 3.3}

(a): Let $\gamma$ be a curve with constant geodesic curvature $\kappa \neq 0$ in any of the three previous constant curvature surfaces. Then, for a given uniform magnetic field $F=\mu \Omega_{2}$, a suitable fitting of the constant speed (and hence, the energy) of $\gamma$ makes this curve to be a magnetic curve of $F$.

(b): Let $(M, g)$ be again one of the three above space forms and $F=\mu \Omega_{2}$ a uniform magnetic field on $(M, g)$. Then, any magnetic curve $\gamma$ with energy $e$ of $(M, g, F)$ can be then considered as a normal magnetic curve of $(M,(1 / e) g,(1 / e) F)$ (see the comment previous to Proposition 2.1).

\section{The Landau-Hall problem in a surface of revo- lution}

Let $\alpha(s)=(f(s), h(s)), a<s<b, f(s)>0$, be a parametrization by the arclength of a curve, $\mathrm{C}$, contained in the $\{x z\}$-plane of $\mathbb{R}^{3}$. We rotate $\mathrm{C}$ around the $z$-axis to obtain a surface of revolution, say $M_{\alpha}$, with canonical parametrization in $\mathbb{R}^{3}$

$$
X(s, v)=(f(s) \cos v, f(s) \sin v, h(s)), \quad 0 \leq v \leq 2 \pi .
$$


Of course we consider that $M_{\alpha}$ is endowed with the induced metric $g$ of the Euclidean one of $\mathbb{R}^{3}$.

Each point of $\mathrm{C}$ describes a parallel, $\gamma_{s}$, which can be parametrized by arclength in the following way

$$
\gamma_{s}(t)=\left(f(s) \cos \frac{t}{f(s)}, f(s) \sin \frac{t}{f(s)}, h(s)\right),
$$

where $0 \leq t \leq 2 \pi f(s)$.

The curvature, $\kappa_{s}$, of $\gamma_{s}$ in $M_{\alpha}$, is computed to be

$$
\kappa_{s}(t)=\left\|\nabla_{T_{s}} T_{s}\right\|=\frac{f^{\prime}(s)}{f(s)}
$$

where $T_{s}=\gamma_{s}^{\prime}$ and $\nabla$ is the Levi-Civita connection of $M_{\alpha}$. In particular, $\kappa_{s}$ is constant along $\gamma_{s}$, and so this curve is a good candidate to be a flowline of a suitable uniform magnetic field on $M_{\alpha}$.

Let $F=\mu \Omega_{2}$ be a uniform magnetic field on $M_{\alpha}$ with constant strength $\mu$. Then $\gamma_{s}$ is a normal magnetic flowline of $\left(M_{\alpha}, g, F=\mu \Omega_{2}\right)$ if and only if $\kappa_{s}=\mu$ (Proposition 3.2). Therefore, the set of magnetic parallels of $\left(M_{\alpha}, g, F=\mu \Omega_{2}\right)$ can be identified with the following subset of the interval $(a, b)$

$$
\Gamma_{\mu}=\left\{s \in(a, b): f^{\prime}(s)=\mu f(s)\right\} .
$$

To determine those surfaces of revolution whose parallels are all normal magnetic curves of a given uniform magnetic field (that is, those with $\Gamma_{\mu}=(a, b)$ ) we need to solve the ordinary differential equation

$$
f^{\prime}(s)=\mu f(s)
$$

Obviously, we have two possibilities. The trivial one, corresponding with the case of a trivial magnetic field (the strength vanishes), the flowlines are then geodesics, and the surface of revolution is a right circular cylinder. Otherwise, since the Gauss curvature of a surface of revolution (in the canonical parametrization) is given by

$$
G(s, t)=-\frac{f^{\prime \prime}(s)}{f(s)},
$$

we get that $G(s, t)=-\mu^{2}$, an hence the surface has constant negative curvature. In particular, we have,

Proposition 4.1 The parallels of a surface of revolution, $M_{\alpha}$, are all normal magnetic flowlines of a uniform magnetic field, $F=\mu \Omega_{2}$, if and only if either:

1. $M_{\alpha}$ is a right circular cylinder (when $\mu=0$ ), or

2. $M_{\alpha}$ is a bugle surface with Gaussian curvature $-\mu^{2}$. 
Let us consider the torus of revolution, $\mathbf{T}(r, R)$, obtained by rotating the circle, $\mathbf{C}$, centered at $(R, 0,0)$ and with radius $r,(R>r)$, around the $z$-axis. The circle can be arclength parametrized by $\alpha(s)=(R+r \cos (s / r), 0, r \sin (s / r))$. Therefore, $f(s)=R+r \cos (s / r)$, with $0 \leq s \leq 2 \pi r$.

Given a uniform magnetic field $F=\mu \Omega_{2}$ on $\mathbf{T}(r, R)$, the set of normal magnetic parallels is identified to

$$
\Gamma_{\mu}=\left\{s \in[0,2 \pi r]: H_{\mu}(s)=0\right\},
$$

where

$$
H_{\mu}(s)=R \mu+r \mu \cos \left(\frac{s}{r}\right)+\sin \left(\frac{s}{r}\right) .
$$

To study $\Gamma_{\mu}=H_{\mu}^{-1}(0)$, we use elemental calculus. First, we assume that $\mu \neq 0$, otherwise $\Gamma_{0}=H_{0}^{-1}(0)$ is made up of the two parallels that are geodesics in $\mathbf{T}(r, R)$. In this setting, we see that $H_{\mu}$ has exactly two critical points on $\mathbf{C}$ (the maximum and the minimum) which are antipodal. In fact, $H_{\mu}^{\prime}(s)=0$ if and only if $\cot (s / r)=r \mu$ and it happens just in the following two antipodal points

$$
\begin{aligned}
& p_{\mu}=\left(\cos \frac{s}{r}, \sin \frac{s}{r}\right)=\left(\frac{r \mu}{\sqrt{1+r^{2} \mu^{2}}}, \frac{1}{\sqrt{1+r^{2} \mu^{2}}}\right), \\
& q_{\mu}=\left(\cos \frac{s}{r}, \sin \frac{s}{r}\right)=-\left(\frac{r \mu}{\sqrt{1+r^{2} \mu^{2}}}, \frac{1}{\sqrt{1+r^{2} \mu^{2}}}\right),
\end{aligned}
$$

The values of $H_{\mu}$ in $p_{\mu}$ and $q_{\mu}$ are

$$
\begin{array}{lll}
H_{\mu}\left(p_{\mu}\right)=R \mu+\sqrt{1+r^{2} \mu^{2}} ; & \text { the maximum of } H_{\mu}, \\
H_{\mu}\left(q_{\mu}\right)=R \mu-\sqrt{1+r^{2} \mu^{2}} ; & \text { the minimum of } H_{\mu} .
\end{array}
$$

We call $D_{\mu}$ the diameter in $\mathbf{C}$ determined by $p_{\mu}$ and $q_{\mu}$, and let $\rho=\left(R^{2}-r^{2}\right)^{-1 / 2}$. We distinguish two cases.

(A) If $\mu>0$, then the point $p_{\mu}$, where $H_{\mu}$ gets its maximum, lies in the first quadrant of the circle C. Since $H_{\mu}\left(p_{\mu}\right)>0$, then $\Gamma_{\mu} \neq \emptyset$ if and only if $H_{\mu}\left(q_{\mu}\right) \leq 0$ (the minimum is non positive) that is $\mu \leq \rho$. Certainly if the equality holds, then $H_{\rho}$ vanishes only at $q_{\rho}=(-r / R,-1 /(\rho R))$. Otherwise, $H_{\mu}$ vanishes exactly in two points, say $z_{\mu}$ and $w_{\mu}$, which are separated by $D_{\mu}$ so they lie in different half circles.

(B) If $\mu<0$, then the point $p_{\mu}$, where $H_{\mu}$ gets its maximum, lies in the second quadrant of the circle $\mathbf{C}$. Since the minimum is negative, $H_{\mu}\left(q_{\mu}\right)<0$, then $\Gamma_{\mu} \neq \emptyset$ if and only if $H_{\mu}\left(p_{\mu}\right) \geq 0$ and it happens if and only if $\mu \geq-\rho$. It is clear that when the equality holds, then $H_{\rho}$ vanishes just at $p_{\rho}=(-r / R, 1 /(\rho R))$. However, if $\mu>-\rho$, then $H_{\mu}$ has exactly two zeroes, say $z_{\mu}^{\prime}$ and $w_{\mu}^{\prime}$, which are obviously separated by $D_{\mu}$ so they lie in different half circles.

All this information can be summarized in the following statement, 
Proposition 4.2 Let $F=\mu \Omega_{2}$ be a uniform magnetic field on a torus of revolution, $\mathbf{T}(r, R)$. Then $\left(\mathbf{T}(r, R), g, \mu \Omega_{2}\right)$ has normal magnetic parallels if and only if $\mu \in[-\rho, \rho]$. Furthermore,

1. If $\mu=-\rho$, then there is one normal magnetic parallel obtained by rotating the point $\left(R+\left(r^{2} / R\right), r /(\rho R)\right) \in \mathbf{C}$.

2. If $\mu=\rho$, then there is one normal magnetic parallel obtained by rotating the point $\left(R-\left(r^{2} / R\right),-r /(\rho R)\right) \in \mathbf{C}$.

3. If $\mu \in(-\rho, \rho)$, then $H_{\mu}$ has exactly two normal magnetic parallels obtained by rotating two points of $\mathbf{C}$ that are separated by $D_{\mu}$.

Example 4.3 Let $C$ be the cathenoid generated by revolving the cathenary curve $\alpha(t)=(\cosh t, 0, t), t \in \mathbb{R}$ around the $z$-axis. Let $F$ be a uniform magnetic field with constant strength $\mu \neq 0$, defined on $C$. Then, it can be shown that there exist magnetic parallels if and only if $\mu \in[-1 / 2,1 / 2]$. Moreover, we have:

1. If $\mu=1 / 2$ (resp. $\mu=-1 / 2$ ), there exists only a normal magnetic parallel corresponding to $t=\ln (1+\sqrt{2})($ resp. $t=-\ln (1+\sqrt{2})$ ).

2. If $\mu \in(-1 / 2,0)$ (resp. $\mu \in(0,1 / 2))$, then $C$ has two normal magnetic parallels which are located in the region $t>0$ (resp. $t<0)$.

Example 4.4 Let $S$ be the revolution surface obtained by rotating the cycloid curve $\alpha(t)=(a(1-\cos t), 0, a(t-\sin t)), t \in(0,2 \pi)$ around the $z$-axis. It is easy to see:

1. If $\mu>0$, then $S$ has only a normal magnetic parallel corresponding to the parameter value $t_{0}=2 \arccos \left[\left(-1+\sqrt{1+16 a^{2} \mu^{2}}\right) /(4 a \mu)\right]$.

2. If $\mu<0$, then $S$ has also only a normal magnetic parallel corresponding to $t_{1}=2 \pi-t_{0}$.

Example 4.5 Finally, let us consider the cone $M$ generated by the line $\alpha(t)=$ $(a t, 0, b t), a^{2}+b^{2}=1, t>0$ around the $z$-axis. Then, it can be seen that for any $\mu>0$, there exists a unique normal magnetic parallel given by $t=1 / \mu$.

\section{Relativistic particles with rigidity of order one}

The search for Lagrangians describing spinning particles (both massive and massless) has a long history. An interesting and unconventional approach is to provide the necessary extra degrees of freedom by actions whose densities depend on higher order geometrical invariants. In particular, this means that those extra bosonic variables must be encoded in the geometry of the world trajectories. The simplest models are those involving density Lagrangians that depend on the curvature, $\kappa$, of the worldlines ([17]-19, etc.). In particular, actions that depend linearly from $\kappa(2],[18-22$, etc.) will be considered in this section. These models describing a massive relativistic boson [3]. 
Suppose that $\Lambda$ is a suitable space of curves (closed curves or clamped curves, for instance) in a Riemannian surface $(M, g)$. Define a one-parameter family of functionals $\mathcal{F}_{m}: \Lambda \rightarrow \mathbb{R}, m \in \mathbb{R}$, by

$$
\mathcal{F}_{m}(\gamma)=\int_{\gamma}(\kappa+m) d s
$$

where $s$ stands for the arclength parameter of curves $\gamma \in \Lambda$. In order to obtain the first variation of these actions, we use the following standard machinery (see for instance [20]). For a curve $\gamma:[0, L] \rightarrow M$, we take variations $\Theta=\Theta(t, r)$ : $[0, L] \times(-\varepsilon, \varepsilon) \rightarrow M$ with $\Theta(t, 0)=\gamma(t)$. Then, we have the variation vector field $W=W(t)=(\partial \Theta / \partial r)(t, 0)$ along the curve $\gamma$. We also put $V=V(t, r)=$ $(\partial \Theta / \partial r)(t, r), W=W(t, r), v=v(t, r)=\|V(t, r)\|, T=T(t, r), N=N(t, r)$, with the obvious meanings. The corresponding reparametrizations will be denoted by $V(s, r), W(s, r)$ etc. The variations of $v$ and $\kappa$ in $\gamma$, in the direction of $W$, can be obtained to be

$$
\begin{gathered}
W(v)=g\left(\nabla_{T} W, T\right) v, \\
W(\kappa)=g\left(\nabla_{T}^{2} W, N\right)-2 g\left(\nabla_{T} W, T\right) \kappa+G g(W, N),
\end{gathered}
$$

here $G$ denotes the Gauss curvature of $(M, g)$ and $\nabla$ its Levi-Civita connection.

Now, we use a standard argument that involves the above formulas so as some integrations by parts to obtain the first derivative of $\mathcal{F}_{m}$

$$
\delta \mathcal{F}_{m}(\gamma)[W]=\int_{\gamma} g(\Omega(\gamma), W) d s+[\mathcal{B}(\gamma, W)]_{0}^{L},
$$

where $\Omega(\gamma)$ and $\mathcal{B}(\gamma, W)$ denote the Euler-Lagrange and the boundary operators and they are respectively given by

$$
\begin{aligned}
\Omega(\gamma) & =(G-m \kappa) N \\
\mathcal{B}(\gamma, W) & =g\left(\nabla_{T} W, N\right)+m g(W, T)
\end{aligned}
$$

Proposition 5.1 (Clamped curves) Given points $q_{1}, q_{2} \in M$ and unit vectors $x_{1} \in$ $T_{q_{1}} M$ and $x_{2} \in T_{q_{2}} M$, define the space of curves

$$
\Lambda=\left\{\gamma:\left[t_{1}, t_{2}\right] \rightarrow M: \gamma\left(t_{i}\right)=q_{i}, T\left(t_{i}\right)=x_{i}, N\left(t_{i}\right)=J x_{i}, 1 \leq i \leq 2\right\}
$$

Then, the critical points of the functional $\mathcal{F}_{m}: \Lambda \rightarrow \mathbb{R}$ are characterized by the following Euler-Lagrange equation

$$
\left.G\right|_{\gamma}=m \kappa,
$$

where $\left.G\right|_{\gamma}$ denotes the Gauss curvature of $(M, g)$ along $\gamma$. 
Proof. Let $\gamma \in \Lambda$ and $W \in T_{\gamma} \Lambda$, then $W$ defines a curve in $\Lambda$ associated with a variation $\Theta$ of $\gamma$. We can perform the following computations along $\Theta$

$$
\begin{aligned}
W & =d \Theta\left(\partial_{r}\right), \\
\nabla_{T} W & =f T+d \Theta\left(\partial_{r} T\right),
\end{aligned}
$$

where $f=\partial_{r}(\ln v)$. We evaluate these formulas along $\gamma$ by making $r=0$ and use that $\Theta$ is a curve in the space $\Lambda$ to obtain

$$
\begin{aligned}
W\left(t_{i}\right) & =0, \\
\nabla_{T} W\left(t_{i}\right) & =f\left(t_{i}\right) x_{i} .
\end{aligned}
$$

As a consequence, $[\mathcal{B}(\gamma, W)]_{t_{1}}^{t_{2}}=0$. So, using Eq. (10), we have that $\gamma$ is a critical point of $\mathcal{F}_{m}: \Lambda \rightarrow \mathbb{R}$, that is $\delta \mathcal{F}_{m}(\gamma)[W]=0$ for any $W \in T_{\gamma} \Lambda$, if and only if $\Omega(\gamma)=0$ which proves the statement.

Similarly, we can obtain the following

Proposition 5.2 (Closed curves) Let $\mathcal{C}$ be the space of immersed closed curves in $(M, g)$. The critical points of the functional $\mathcal{F}_{m}: \mathcal{C} \rightarrow \mathbb{R}$ are those closed curves that are solutions of the following Euler-Lagrange equation

$$
\left.G\right|_{\gamma}=m \kappa .
$$

\section{Gaussian magnetic fields}

Let $M$ be a surface immersed in the Euclidean three-space, $\mathbb{R}^{3}$, so the metric, $g$ is the induced one. We denote by $N: M \longrightarrow \mathbb{S}^{2}$ its Gauss map and $d \sigma^{2}$ will stand for the area element on the unit round sphere $\mathbb{S}^{2}$. The two form $N^{*}\left(d \sigma^{2}\right)$ on $M$ can be used, for example, to measure areas of the spherical images or topological total charges of solitons in the $O(3)$ non-linear sigma model (see for instance [23], 24] and references therein). In this section we will consider magnetic fields of the type

$$
F=\frac{1}{m} N^{*}\left(d \sigma^{2}\right),
$$

where $m$ is a non zero constant. We call then Gaussian magnetic fields (GMF). It is well known that $N^{*}\left(d \sigma^{2}\right)=G \Omega_{2}, G$ denoting the Gaussian curvature of $(M, g)$ and this, in particular, implies that we can consider these kind of magnetic fields with no mention to the surrounding space. Namely, a GMF is always of the type

$$
F=\frac{G}{m} \Omega_{2} .
$$

The Lorentz force of a GMF is computed to be $\Phi=(G / m) J$, where $J$ is the standard complex structure in $M$. In particular, for any unit vector field, $X$, on $M$, the matrix of $\Phi$, in the terminology of Section 3, with respect to an orthonormal frame $\{X, J X\}$ is given by 


$$
\left(\begin{array}{rr}
0 & -\frac{G}{m} \\
\frac{G}{m} & 0
\end{array}\right) .
$$

In this framework, we can combine Proposition 3.1 and the field equations of the particle models defined from $\mathcal{F}_{m}$, see Eq. (7), to obtain the following amazing relationship between the flow of a GMF and the worldline trajectories of relativistic particles with order one. To be precise, we have,

Theorem 6.1 Let $\gamma \in \Lambda$ be a curve (clamped or closed) in $(M, g)$. Then it is a normal flowline of $\left(M, g, F=(G / m) \Omega_{2}\right)$ if and only if it is a critical point (world line) of the action $\mathcal{F}_{m}: \Lambda \rightarrow \mathbb{R}$ given by

$$
\mathcal{F}_{m}(\gamma)=\int_{\gamma}(\kappa+m) d s
$$

At this point, we can take advantage of the variational approach to study stability of the GMF flowlines. Therefore, we need the second derivative of $\mathcal{F}_{m}$ in a critical point, say $\gamma$ in a suitable space of curves, $\Lambda$ (recall closed or clamped curves). After some long computations (see [17] for details) one can obtain the following expression

$$
\delta^{2} \mathcal{F}_{m}(\gamma)[W]=\left.\int_{\gamma} g\left(W, \nabla_{W} \Psi\right)\right|_{\gamma} d s
$$

where $\Psi$ denotes the vector field, along a variation of $\gamma$, given by $\Psi=(G-m \kappa) N$. Now, we choose $W=\Phi N$ to obtain

$$
\left.g\left(W, \nabla_{W} \Psi\right)\right|_{\gamma}=\Phi^{2} N(G-m \kappa),
$$

where the right hand term is restricted to $\gamma$. However the variation of $\kappa$ was given in Eq. (9); so, in particular, we have $N(\kappa)=\kappa^{2}+G$. Then, one gets from Eq. (12)

$$
\delta^{2} \mathcal{F}_{m}(\gamma)[W]=\int_{\gamma} \Phi^{2}\left(N(G)-\frac{1}{m}\left(G^{2}+m^{2} G\right)\right) d s .
$$

Hence, we have the following useful test of stability.

Proposition 6.2 A critical point, $\gamma \in \Lambda$, of $\mathcal{F}_{m}$ is stable if and only if the function $N(G)-(1 / m)\left(G^{2}+m^{2} G\right)$ is signed along $\gamma$.

It should be observed that the previous test has the following geometrical meaning. Put $\varphi=G-m \kappa$, then $\gamma \subset \varphi^{-1}(0)$, because it is a critical point of $\mathcal{F}_{m}$, and then stability means that $\gamma$ is made up of regular points of $\varphi$. Moreover, observe that this happens if 0 is a regular value of $\varphi$.

Now, let us use all this information in the following elemental setting. We consider $M=\mathbb{S}^{2}(1)$ the unit round sphere. Then any GMF, $F=(G / m) \Omega_{2}=(1 / m) \Omega_{2}$, is uniform. However, when studying uniform magnetic fields on a round sphere, we can not talk about stability of magnetic trajectories. This is not the case of our approach. 
In this setting, the magnetic curves are, according Theorem 6.1, the critical points of the functional

$$
\mathcal{F}_{m}(\gamma)=\int_{\gamma}(\kappa+m) d s=\int_{\gamma} \kappa d s+m L(\gamma)
$$

and they are nothing but those curves that satisfy $1=m \kappa$, that is geodesic circles with geodesic curvature $\kappa=1 / m$.

On the other hand, we can use the Gauss-Bonnet formula to see that this variational problem is equivalent to that associated with the action $\mathcal{D}_{m}: \mathbf{D} \longrightarrow \mathbb{R}$ defined by

$$
\mathcal{D}_{m}(\Delta)=\int_{\Delta} G \Omega_{2}+m \int_{\partial \Delta} d s=\operatorname{Area}(\Delta)+m L(\partial \Delta),
$$

acting on the space $(D)$ of simply-connected domains, $\Delta$ in $\mathbb{S}^{2}$, with the same boundary $\gamma=\partial \Delta$. This is nothing but the isoperimetric problem in the round sphere. The solution is a couple of domains $\Delta_{1}$ and $\Delta_{2}$ (the maximum and the minimum) with common boundary a geodesic circle, $\gamma$, of curvature $\kappa=1 / m$. Since $N(G)=0$ then, $-\left(1+m^{2}\right) / m$ has obviously sign for any choice of the coupling constant $m$. Consequently, calling to Proposition 6.2 the solutions are stable.

The case where $m=0$ deserves a few words. First of all GMF with $m=0$ could be considered as a limiting case, however, after our variational approach, it can be identified with the massless Plyushchay model, [21, which is governed by the Lagrangian

$$
\mathcal{F}_{0}(\gamma)=\int_{\gamma} \kappa d s
$$

This model has been considered with detail in [18]. For example the sphere does not admit, non only minima (maxima) for this model but also critical points. However, an anchor ring has two critical points corresponding to the two parallels of parabolic points.

\section{GMF flowlines on some non-constant Gauss cur- vature surfaces}

In this last section we would like to analyze when certain relevant curves on some non-constant Gauss curvature surfaces are in fact magnetic.

We recalled the explicit expression of the Gauss curvature, $G(s, v)$, of a surface of revolution, $M_{\alpha}$, see Eq. (6). Consequently, we can assert now that a parallel, $\gamma_{s}$, is a normal flowline of the GMF given by $(G / m) \Omega_{2}$ on $M_{\alpha}$ if and only if

$$
f^{\prime \prime}(s)+m f^{\prime}(s)=0 \text {. }
$$

Next, we will obtain the surfaces of revolution whose parallels are all normal flowline of a GMF. In contrast to the case of a uniform magnetic field, where only the bugle surface appeared as a solution (see Proposition 4.1), now the general solution is 
made up of a three parameter family of surfaces which includes the bugle surface too.

Theorem 7.1 The normal flow of a $G M F,(G / m) \Omega_{2}$, in a surface of revolution, $M_{\alpha}$, is invariant under rotations if and only if the profile curve of $M_{\alpha}$ lies in the following three parameter family of arclength parametrized plane curves

$$
\alpha(s)=\left(f(s), \int_{0}^{s} \sqrt{1-f^{\prime}(s)^{2}} d s\right)
$$

where

$$
f(s)=\frac{1}{m}(a+c \exp (-m s+b)) ; \quad a, b, c \in \mathbb{R} \quad \text { with } \quad a>0 .
$$

Observe that the general solution of the ordinary differential equation (13) has the form $f(s)=(a+c \exp (-m s+b)) / m$, so the proof of the last result becomes obvious. Observe also that the above characterized class of surfaces of revolution includes the bugle surface $(a=0)$ and the right circular cylinder $(c=0)$ too.

It should be noticed the following coupling phenomenon in a surface of revolution, $M_{\alpha}$, between the GMF, $F_{1}=(G / m) \Omega_{2}$ and the uniform magnetic field $F_{2}=-m \Omega_{2}$, for some values of the coupling constant $m$. Suppose, for example, that $M_{\alpha}=$ $\mathbf{T}(r, R)$ is a torus of revolution and $\rho=\left(R^{2}-r^{2}\right)^{-1 / 2}$ (notation as in Section 4). Then, $F_{1}$ always has two parallels being normal magnetic curves, no matter the value of $m$. Now, we use Proposition 4.2 to obtain the following statement,

Proposition 7.2 If $-m \in(\rho, \rho)$, then both $F_{1}$ and $F_{2}$ have two normal magnetic parallels coming from points alternatively placed in the profile circle. Moreover they collapse when $-m$ goes to $-\rho$ or $\rho$.

Proof. For any value of $m$ in $\mathbb{R},\left(\mathbf{T}(r, R), g, F_{1}\right)$ has two normal magnetic parallels obtained by rotation of the two antipodal points in $\mathbf{C}$, defined by $\cot (s / r)=-r m$. These two points are just those determining the diameter $D_{-m}$ that separates the two magnetic parallel of $\left(\mathbf{T}(r, R), g, F_{2}\right)$ when $-m \in(-\rho, \rho)$. The second part of this statement follows similarly when use points 1 and 2 of Proposition 4.2 .

We finish the paper showing several examples.

Example 7.3 Let $\beta(s)$ be an arclength parametrized curve contained in a plane, $\Pi$ (with unit normal vector $B_{0}$ ), in $\mathbb{R}^{3}$. We denote by $\{T(s), N(s)\}$ a Frenet frame along $\beta(s)$, so that $T(s) \wedge N(s)=B_{0}$, and $\kappa(s)$ will stand for its curvature function. For a suitable $r>0$, we define a tube of radius $r$, say $\mathrm{T}_{\beta}(r)$, as the surface given by

$$
X(s, v)=\beta(s)+r\left(\cos (v) N(s)+\sin (v) B_{0}\right) .
$$

We denote by $\Lambda_{\beta}=\left\{\gamma_{v},: v \in[0,2 \pi]\right\}$ the family of curves in the tube obtained when we make $v$ constant. The curvature of these curves in $\mathrm{T}_{\beta}(r)$ can be obtained, from a direct computation, to be 


$$
\kappa_{v}(s)=\frac{\kappa(s) \sin (v)}{1-r \kappa(s) \cos (v)} .
$$

Notice that it is not constant unless $\beta(s)$ is chosen to be constant curvature.

On the other hand, the Gauss curvature of the tube $\mathrm{T}_{\beta}(r)$ is computed to be

$$
G(s, v)=-\frac{\kappa(s) \cos (v)}{r(1-r \kappa(s) \cos (v))} .
$$

Now, we can apply these formulas together with the Euler-Lagrange equations associated with the GMF, $F=(G / m) \Omega_{2}$ (Propositions 5.1, 5.2), to see that there exist exactly two curves (clamped or closed) in $\Lambda_{\beta}$ that are normal magnetic trajectories. They are obtained for $\cot (v)=-r m$ and this is, formally, the same result that we have obtained for a torus of revolution (Proposition 4.2) which can be regarded as a tube around a circle.

Example 7.4 Similarly, for a curve, $\beta(s)$, in $\mathbb{R}^{3}$ with Frenet frame $\{T(s), N(s), B(s)\}$, curvature $\kappa(s)$ and torsion $\tau(s)$, one can define the tube $\mathrm{T}_{\beta}(r)$ by

$$
X(s, v)=\beta(s)+r(\cos (v) N(s)+\sin (v) B(s)) .
$$

In particular, if $\beta(s)$ is a helix ( $\kappa$ and $\tau$ are both constant) then the curvature function, $\kappa_{v}(s)$ of the curves in $\Lambda_{\beta}=\left\{\gamma_{v},: v \in[0,2 \pi]\right\}$ satisfy

$$
\kappa_{v}^{2}=\frac{\kappa^{2} \sin ^{2}(v)}{(1-r \kappa \cos (v))^{2}+r^{2} \tau^{2}} .
$$

Now, the curves in $\Lambda_{\beta}$ that are normal flowlines of $(G / m) \Omega_{2}$ on the helicoidal tube $\mathrm{T}_{\beta}(r)$ correspond with the zeroes of the function $\vartheta: \mathbb{S}^{1} \rightarrow \mathbb{R}$ defined by

$$
\vartheta(v)=(1-r \kappa \cos (v))^{2}\left(\cos ^{2}(v)-r^{2} m^{2} \sin ^{2}(v)\right)+r^{2} \tau^{2} \cos ^{2}(v) .
$$

However, we have

$$
\vartheta(0)=\vartheta(\pi)=(1-r \kappa)^{2}+r^{2} \tau^{2}>0 \quad \text { and } \quad \vartheta\left(\frac{\pi}{2}\right)=\vartheta\left(\frac{3 \pi}{2}\right)=-r^{2} m^{2}<0 .
$$

Therefore, there exist four curves of $\Lambda_{\beta}$ in the flow of $(G / m) \Omega_{2}$.

Example 7.5 On the cathenoid (Example 4.3), the GMF given by $(G / m) \Omega_{2}$ has a unique normal magnetic parallel for all $m$. If $m>0$, it is obtained for a $t_{0}<0$. If $m<0$, then it is obtained for a $t_{0}^{\prime}=-t_{0}>0$.

Example 7.6 On the hyperboloid of revolution obtained from Eq. (15) by putting $f(t)=\cosh t$ and $h(t)=\sinh t$, the GMF given by $(G / m) \Omega_{2}$ has also a unique normal magnetic parallel for all $m$, analogously to the previous case. 
Example 7.7 On the cicloidal surface (Example 4.4), the GMF given by $(G / m) \Omega_{2}$ has a unique normal magnetic parallel for $m \in(-\infty,-1 /(4 a)) \cup(1 /(4 a), \infty)$. If $m>0, t_{0}=\arccos \left((1 /(4 a m))\right.$, whereas if $m<0$, then $t_{0}^{\prime}=2 \pi-t_{0}$.

\section{Conclusions}

Oriented surfaces, $M$, in $\mathbb{R}^{3}$ admit two natural 2-forms. First, the area element, $\Omega_{2}$, associated with the induced metric, $g$. Second, the area element, $N^{*}\left(d \sigma^{2}\right)$, of its spherical image under the Gauss map, $N: M \rightarrow \mathbb{S}^{2}$. It is well known that these 2 -forms are nicely related by

$$
N^{*}\left(d \sigma^{2}\right)=G \Omega_{2}
$$

where $G$ enotes the Gaussian curvature of $g$. In particular, both 2-forms are intrinsic and then they are defined once we know a Riemannian metric, $g$, on $M$. Associated with these 2 -forms appear two classes of magnetic fields on $(M, g)$,

1. The class made up of the constant multiples of the former one, $\mathcal{C}_{1}=\left\{\mu \Omega_{2}\right.$ : $\mu \in \mathbb{R}\}$, provides that of uniform magnetic fields, with strength $\mu$, on $(M, g)$. The corresponding Landau-Hall problem has been widely studied along the literature. Even in this paper, we have obtained some new information relative to uniform magnetic field essentially in a surface of revolution. For example, we have characterized right circular cylinders and bugle surfaces as the only surfaces of revolution whose parallels are all normal magnetic flowlines of a uniform magnetic fields.

2. The class of the constant multiples of the later one, $\mathcal{C}_{2}=\left\{\mu N^{*}\left(d \sigma_{2}\right): \mu \in \mathbb{R}\right\}$, constitutes a class of magnetic fields that in this paper are introduced under the terminology of Gauss-Landau-Hall magnetic fields (GMF). In this case the strength is given by $\mu G$ and obviously both classes coincide when $(M, g)$ has constant curvature.

In this paper, we wish to state the importance and nice interest of GMF on surfaces. In fact, the chief result of the paper appears when we study the Landau-Hall problem associated with a GMF (which we call the Gauss-Landau-Hall problem). Then, we are be able to show that this problem is equivalent to the dynamics of a massive relativistic boson. This provides an amazing relationship between two, a priori, quite different physical phenomena.

Therefore, we can use two points of view to study each of the two involved problems. On one hand, one can study completeness, homogeneity and so on, in the dynamical study of bosonic worldlines. By the way, we have introduced a section with new results on these topics. But on the other hand, the Gauss-Landau-Hall problem can be regarded as a variational problem globally stated. In this setting, flowlines are critical points of an action which has been used to model relativistic particles with order one rigidity. In particular, we can talk about, and so we study, global stability of normal flowlines of a GMF. Say finally that under this equivalence, 
the model to describe a massless particle with arbitrary helicity correspond with a limit case obtained when the force of the GMF increases arbitrarily.

We believe that this new point of view in the study of GMF is physically remarkable and it could be extended to other classes of magnetic fields.

\section{Acknowledgments}

Research partially supported by MCYT FEDER Grant BFM 2001-2871-C04 and by Acción Coordinada Grupos de Investigación FQM-324 and FQM-327, Junta de Andalucía.

\section{References}

[1] L.D. Landau and E. M. Lifschitz, Course of Theoretical Physics, Vol. 1. Mechanics. 3rd edition. (Pergamon Press, Oxford-New York-Toronto, 1976).

[2] M. S. Plyushchay, Phys. Lett. B, 243, 383 (1990).

[3] M.S. Plyushchay, Int. J. Mod. Phys. A, 4, 3851 (1989).

[4] R. K. Sachs and H. Wu, General Relativity for mathematicians, in Graduate texts in Mathematics, no.48, (Springer-Verlag, New York, 1977).

[5] A. Romero and M. Sánchez, "Completeness of the solutions of certain differential equation on semi-Riemannian manifolds: Motion of particles on a force field" (Univ. Granada, umpublished).

[6] R. Abraham and J. E. Marsden, Foundations of Mechanics, 2nd. Ed. (Perseus Books, Massachusetts, 1988).

[7] B. O'Neill, Semi-Riemannian geometry with applications to Relativity (Academic Press, New York, 1983).

[8] A. Lopez-Almorox, Publ. R. Soc. Mat. Esp., vol. 3, 133 (2001).

[9] K. Nomizu and H. Ozeki, Proc. Amer. Math. Soc., 12, 889 (1961).

[10] T. Adachi, Tsukuba J. Math., 20, 225 (1996).

[11] A. Comtet, Ann. Phys., 173, 185 (1987).

[12] N. Gouda, Tôhoku Math. J., 49, 165 (1997); J. Math. Soc. Japan, 50, 767 (1998).

[13] D. A. Kalinin, Rep. Math. Phys., 39, 299 (1997).

[14] A. Lopez-Almorox and C. Tejero-Prieto, Rev. R. Acad. Cienc. Exactas Fís. Nat. Ser. A Mat., 95no. 2, 259 (2001).

[15] G.P. Paternain and M. Paternain, Nonlinearity, 10, 121 (1997). 
[16] M. P. Wojtkowski, Fund. Math., 163, 177 (2000).

[17] J. Arroyo, Presión calibrada total. (PhD Thesis, UPV, 2001).

[18] J. Arroyo, M. Barros and O.J. Garay, J. Geom. Phys., 41, 65 (2002).

[19] V. V. Nesterenko, A. Feoli and G. Scarpetta, J. Math. Phys., 36, 5552 (1995); Class. Quant. Grav., 13, 1201 (1996).

[20] M. Barros, Gen. Relativity Gravitation, 34, 837 (2002).

[21] M. S. Plyushchay, Mod. Phys. Lett., 4, 837 (1989).

[22] M. S. Plyushchay, "Comment on the relativistic particle with curvature and torsion of world trajectory," hep-th/9810101.

[23] M. S. Ody and L. H. Ryder, Int. J. Mod. Phys. A, 10, 337 (1995).

[24] T. Tsurumaru and I. Tsutsui, Phys. Lett. B, 460, 94 (1999). 